\title{
Molecular AND-logic for dually controlled activation of a DNA-binding spiropyran $\dagger$
}

\author{
Martin Hammarson, Johanna Andersson, Shiming Li, Per Lincoln and Joakim Andréasson*
}

Received 1st June 2010, Accepted 5th August 2010

DOI: $10.1039 / \mathbf{c 0 c c 0 1 6 8 2 a}$

A spiropyran photoswitch is activated using UV light and protons from a form that shows no interaction with DNA to a form that binds to DNA by intercalation. This scheme is interpreted as a biologically relevant logic AND gate with potential applications as a dually controlled anticancer drug.

Molecular logic has traditionally been used in the mimicry of the functions performed by silicon-based electronic devices. ${ }^{1-5}$ Here, cleverly designed decision-making molecules deliver a binary on (1)-off (0) response upon exposure to stimuli such as ions, voltage, and light, according to the respective logic scheme. The entire set of two-input logic gates has been mimicked on the molecular scale, and today, this line of research is targeting advanced logic operations such as the addition, subtraction, multiplexing/demultiplexing, and encoding/decoding of binary digits. ${ }^{6-14}$ It has been argued, however, that walking in the footsteps of the silicon-based predecessors may not lead to the soonest practical applications for molecular logic. ${ }^{15}$ Instead, fields should be identified where semiconductor materials cannot go. Biological systems, e.g., the human body, serve as good examples. Recently, Konry and Walt interpreted a medical diagnostic protocol using the Boolean logic language, ${ }^{16}$ whereas the groups of Zink/Stoddart, Shabat, and Aida have used the logic response from molecules to trigger the activation and the release of (encapsulated) drugs. ${ }^{17-19}$ Dually controlled drug-activation is of specific interest, as it would lead to a better spatiotemporal control and increase the selectivity even further. Ozlem and Akkaya recently described a BODIPY derivative designed for dually controlled photodynamic therapy, using the logic AND gate as the template. ${ }^{15} \mathrm{H}^{+}$and $\mathrm{Na}^{+}$ions served as the inputs, whereas efficient generation of toxic singlet oxygen represented the output. The inspiration for the study is the fact that the concentration of both $\mathrm{H}^{+}$and $\mathrm{Na}^{+}$is drastically increased in cancerous tissue. ${ }^{20-22}$ Another frequently used method for anticancer treatment is to use a drug that binds very strongly to DNA. ${ }^{23}$ In this way, proliferation is inhibited, and the cells die. The downside with this approach is the very low discrimination between cancer cells and normal cells, leading to severe side effects. Therefore, controlling the DNA-binding action using external and/or internal stimuli would be of tremendous utility. ${ }^{24}$ Previous activities in our laboratory have focused on activation of spiropyran (SP) photoswitches using

Department of Chemical and Biological Engineering, Physical Chemistry, Chalmers University of Technology, 41296 Göteborg, Sweden.E-mail: a-son@chalmers.se; Fax: +46 3177238 58; Tel: +46317722838

$\dagger$ Electronic supplementary information (ESI) available: Synthesis, experimental section and details on the DNA-binding. See DOI: $10.1039 / \mathrm{c} 0 \mathrm{cc} 01682 \mathrm{a}$
UV light as the only stimulus. ${ }^{25}$ Here, we report a photochromic SP derivative that is activated by the combined application of UV light together with $\mathrm{H}^{+}$from a form that does not display any DNA-binding properties to a form that binds to DNA by intercalation. Thus, it is interpreted as a biologically relevant AND logic gate driven by UV light and $\mathrm{H}^{+}$as inputs and DNA-binding (potential cytotoxicity) as output. $\mathrm{H}^{+}$could be considered as an internal, or sensory, input and the choice is motivated by the fact that the $\mathrm{pH}$ value in cancer cells is significantly lower compared to healthy cells, ${ }^{21,22}$ whereas the UV light is an externally controlled input.

Scheme 1 shows the structures and the isomerization scheme of SP 1 used in this study. The thermally stable spiro form $\mathbf{1 c}$ is isomerized to the open merocyanine form 10 using UV light, and the reverse process is stimulated by visible light exposure. The photostationary distribution after exposure to $254 \mathrm{~nm}$ UV light is ca. $65 / 35$ 1c/1o, whereas visible light $(600>\lambda>475 \mathrm{~nm}$, $\sim 10 \mathrm{~mW} \mathrm{~cm}^{-1}$ ) converts the sample to virtually $100 \%$ 1c with a time constant of $\sim 3 \mathrm{~min}$. Left in the dark, a 90/10 1c/1o thermal equilibrium is established with a time constant of $75 \mathrm{~min}$ at $20{ }^{\circ} \mathrm{C}$. Hydrolysis ${ }^{26}$ of 10 is also observed in the dark, albeit at a much slower rate (time constant $\sim 80$ hours). The formation of $\mathbf{1 o H}^{+}$is stimulated by acidification of $\mathbf{1 0}$ $\left(\mathrm{p} K_{\mathrm{a}}=4.5\right)$, and the reaction is reversed by addition of base. $\mathbf{1 0 H}^{+}$may also be formed from $\mathbf{1 c}$ in acidic environment, although at a much slower rate compared to the thermal opening. Thus, this process does not contribute significantly to the formation of $\mathbf{1 o H ^ { + }}$. Furthermore, there is no thermal conversion from $\mathbf{1 o H} \mathbf{H}^{+}$to $\mathbf{1 c}$, although the same process is photoinduced by visible light exposure. As shown in Fig. 1, 1c (blue line) absorbs exclusively in the UV region with absorption maxima at 238 and $286 \mathrm{~nm}$. $\mathbf{1 o}$ (red line) and $\mathbf{1 0 \mathbf { H } ^ { + }}$ (green line) display absorption also in the visible region with the most intense peaks at 515 and $416 \mathrm{~nm}$, respectively.

DNA-binding typically manifests itself in changes in the absorption spectrum of the interacting molecule. The absorption spectra of 1c with and without the addition of calf thymus

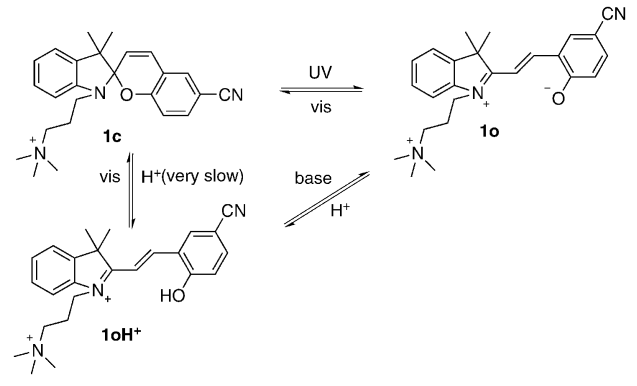

Scheme 1 Structures of the closed spiro (1c), open merocyanine (1o), and protonated merocyanine $\left(\mathbf{1 o H} \mathbf{H}^{+}\right)$forms of photoswitch $\mathbf{1}$. 


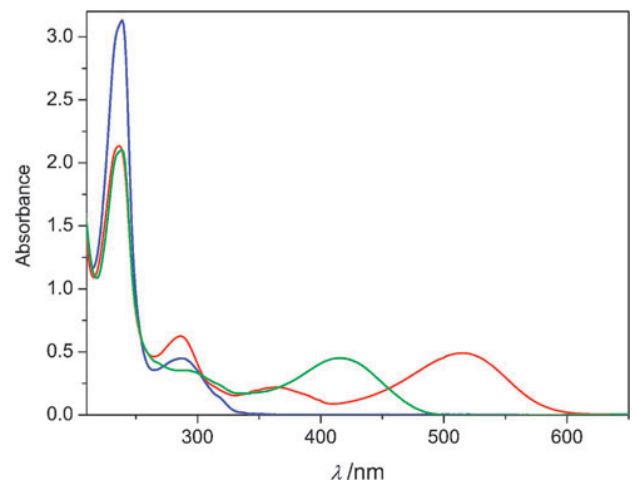

Fig. 1 Absorption spectra of $\mathbf{1}$ in $\mathrm{mQ}$ water before UV irradiation ( $100 \%$ 1c blue line), after $10 \mathrm{~min}$ of $254 \mathrm{~nm}$ UV irradiation (ca. 65/35 1c/1o red line), and after subsequent acidification of the solution ( $\sim \mathrm{pH} 2, c a .65 / 35 \mathbf{1 c} / \mathbf{1 o H}^{+}$green line). The total concentration of 1 was $c a .60 \mu \mathrm{M}$.

(CT) DNA at pH 7 shown in Fig. 2a are virtually superimposable, signalling no significant interactions between $\mathbf{1 c}$ and DNA. The corresponding absorption spectra after UV irradiation are shown in Fig. 2b. Formation of $\mathbf{1 0}$ is confirmed by the absorption band at $515 \mathrm{~nm}$. The similarity between the spectra with and without added DNA, however, indicates that no substantial DNA-binding occurs. The same set of measurements were performed also at $\mathrm{pH} 6,{ }^{27}$ equivalent to the addition of $\mathrm{H}^{+}$, and the results are shown in Fig. $2 \mathrm{c}$ and d. From Fig. $2 \mathrm{c}$ it is clearly seen that 1c (no UV) does not interact significantly with DNA. In sharp contrast, the spectral changes upon addition of DNA after UV irradiation are dramatic, as illustrated in Fig. 2d. The spectrum after addition of DNA has experienced a $c a .50 \%$ decrease at wavelengths longer than $500 \mathrm{~nm}$, where 10 is the only absorber, and also a significant increase between $400 \mathrm{~nm}$ and $450 \mathrm{~nm}$. This strongly suggests that $\mathbf{1}$ binds to DNA after UV irradiation at $\mathrm{pH} 6$, but virtually no binding occurs at any of the other combinations of $\mathrm{pH}$ and UV dose.

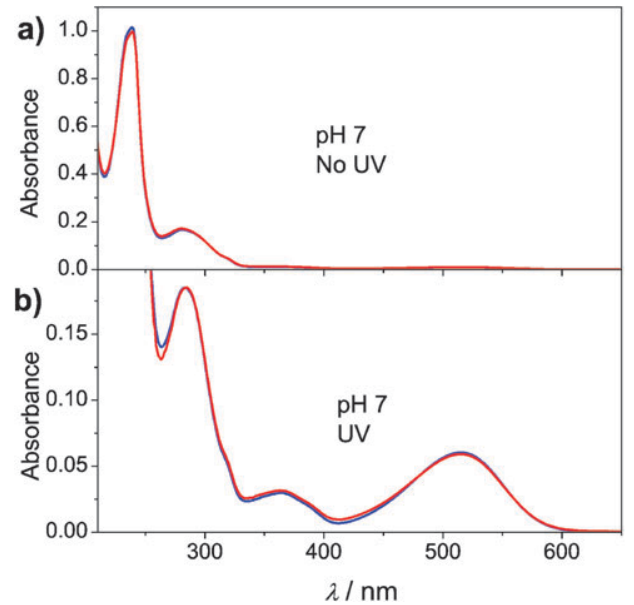

In order to support this notion, flow oriented linear dichroism (LD) measurements ${ }^{28}$ of $\mathbf{1}+\mathrm{CT}$ DNA at $\mathrm{pH} 7$ and 6 were undertaken. Here, the DNA molecules are aligned in a shear flow, so that any LD signal originating from $\mathbf{1}$ is a manifestation of DNA-binding. Fig. 3 shows the LD spectra of equimolar samples of 1 at $\mathrm{pH} 7$ (blue line) and 6 (red line) after $10 \mathrm{~min}$ UV irradiation in the presence of DNA. The negative LD signals indicate binding by intercalation between the DNA-bases. Furthermore, the reduced $\mathrm{LD}(\mathrm{LD} / \mathrm{Abs})$ can be used to determine the angle between the transition moment of the DNA-binding molecule and the DNA helix axis. Here, the angle between the transition of DNA-bound $\mathbf{1}$ centered around $431 \mathrm{~nm}$ (vide infra) and the helix axis is $75 \pm 5^{\circ}$, which confirms the notion of intercalation being the binding mode..$^{25,29,30}$

The amplitude of the LD signal is proportional to the amount of DNA-bound 1, provided a uniform binding geometry. Here, the LD signal is increased by a factor of $\sim 20$ on going from $\mathrm{pH} 7$ to $\mathrm{pH} 6$. The decrease in absorption at $515 \mathrm{~nm}$ upon addition of DNA (see Fig. $2 \mathrm{~b}$ and d) is another measure of the amount of DNA-bound 1, and it is encouraging to note that the absorption change is amplified by a factor of $\sim 25$ at $\mathrm{pH} 6$ compared to $\mathrm{pH} 7$, i.e., there is a very good quantitative agreement between the absorption and the LD measurements. Furthermore, subtracting the contribution from the unbound forms of $\mathbf{1}$ from the absorption spectrum of $1+$ DNA after UV irradiation at $\mathrm{pH} 6$, shown as the red line in Fig. 2d, yields a spectrum with absorption maximum at $431 \mathrm{~nm}$ that virtually coincides with the LD spectrum (see inset in Fig. 3). These results strongly suggest that $\mathbf{1 0 H}{ }^{+}$is the DNAbinding species, as the spectrum of the DNA-binding form is red shifted $c a .14 \mathrm{~nm}$ compared to $\mathbf{1 0 H ^ { + }}$ in solution, which is a typical observation for DNA intercalating dyes. Furthermore, it is well established that protonation facilitates DNA-binding, as the extra positive charge increases the electrostatic attraction to the negatively charged DNA phosphate backbone. $\mathbf{1 0 H ^ { + }}$ is not thermally interconverted to any of the other two forms,

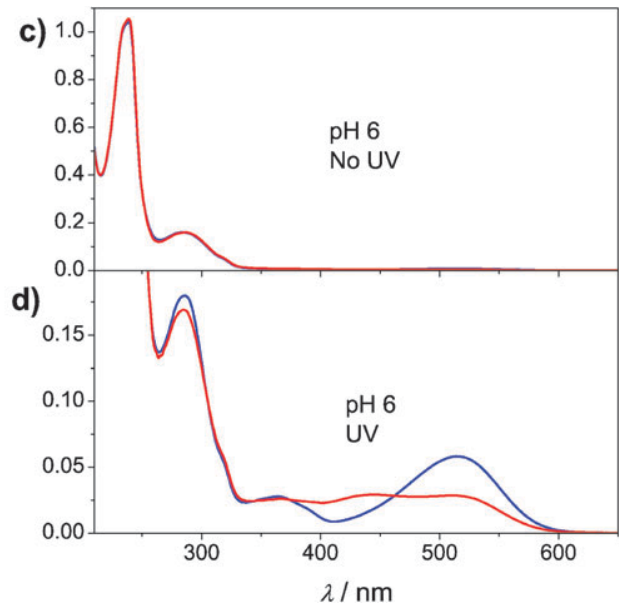

Fig. 2 Absorption spectra of 1 in the absence (blue line) and in the presence (red line) of calf-thymus DNA at pH 7 (a and b) and at pH 6 (c and d). In (b) and (d), the samples were exposed to $c a$. $50 \mathrm{~s}$ UV light to stimulate the light induced isomerization 1c $\rightarrow$ 1o to a final $90 / 10$ distribution $1 \mathbf{c} /\left(\mathbf{1 0}+\mathbf{1 0} \mathbf{H}^{+}\right)$. In (d), it is clearly seen that the spectral changes upon addition of DNA are dramatic at pH 6 after UV irradiation, while no or only minor changes are observed for all other combinations of $\mathrm{pH}$ and UV dose. The contributions from DNA absorption have been subtracted for ease of comparison. The concentrations of $\mathbf{1}$ and DNA were $c a .20 \mu \mathrm{M}$ and $110 \mu \mathrm{M}$, respectively, and the $\mathrm{NaCl}$ concentrations of the solutions were $10 \mathrm{mM}$. Note that different $y$-axis scales have been used for the top and the bottom rows in order to better visualize the effect of DNA on the absorption spectra of the open forms $\mathbf{1 0}$ and $\mathbf{1 0 H ^ { + }}$ which were not formed in the absence of UV exposure (a and c). 


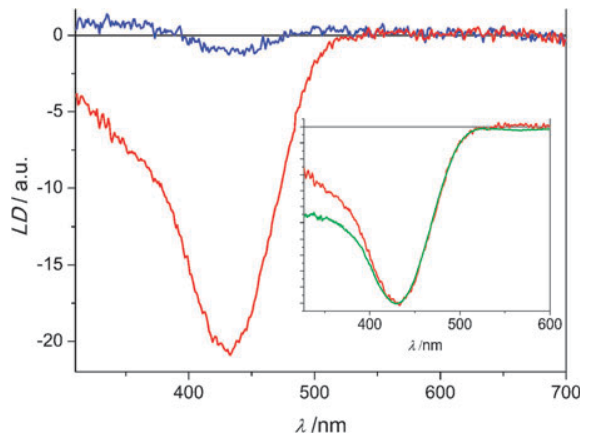

Fig. 3 Flow oriented LD spectra on samples of $\mathbf{1}$ and CT DNA after 10 min UV irradiation at $\mathrm{pH} 7$ (blue line) and at $\mathrm{pH} 6$ (red line). The amplitude of the LD signal is $c a$. 20 times higher at $\mathrm{pH} 6$ compared to $\mathrm{pH}$ 7. The inset shows the good agreement between the LD spectrum at pH 6 (red line) and the absorption spectrum of DNA-bound $\mathbf{1 o H}^{+}$(green line). The negative absorption spectrum is shown for ease of comparison. The concentrations of 1 and DNA were $c a$. $50 \mu \mathrm{M}$ and $90 \mu \mathrm{M}$, respectively, and the $\mathrm{NaCl}$ concentrations of the solutions were $10 \mathrm{mM}$.

which implies that once $\mathbf{1}$ has been activated to the DNAbinding form, it is not deactivated unless external stimuli is applied. Light exposure is such a stimuli ( $c f$. Scheme 1), and it is encouraging to see that the DNA-binding process is fully reversible using visible light irradiation to trigger the dissociation (see the ESI $\dagger$ ).

Inspection of the changes in absorption of $\mathbf{1}$ upon addition of DNA shown in Fig. 2 makes it possible to estimate the DNA-binding constant for $\mathbf{1 o H}^{+}$(see the ESI $\dagger$ for details). This $\mathrm{pH}$-independent microscopic binding constant was determined to $2.9 \times 10^{5} \mathrm{M}^{-1}$. The corresponding macroscopic binding constant for $\mathbf{1}$, however, is dependent on $\mathrm{pH}$ and UV dose. The resulting numbers for the four applied $\mathrm{UV}$ and $\mathrm{pH}$ situations are collected in Table 1. The table is outlined according to the truth table for a Boolean logic gate with two binary inputs and one binary output. The variation of the binding constant with input combinations observed in Table 1 is referred to as logic AND gate behavior, i.e., both the UV input and the $\mathrm{H}^{+}$input must be applied (set to 1) in order to observe a high output signal, here represented by a high binding constant. All other input combinations yield a low output.

The $\mathrm{pH}$ value may differ substantially between different types of tumors. Hence, it would be of high utility to be able to control the $\mathrm{pH}$ at which the drug is being protonated, i.e., by fine-tuning the $\mathrm{p} K_{\mathrm{a}}$-value, to differentiate between normal cells and cancer cells. The $\mathrm{p} K_{\mathrm{a}}$-value of the phenolate oxygen of spiropyran photoswitches depends on the electron withdrawing properties of the group in para-position, which allows for

Table 1 Truth table for the AND logic gate using UV light and $\mathrm{H}^{+}$ as inputs and the macroscopic DNA-binding constant of $\mathbf{1}$ as output

\begin{tabular}{lcc}
\hline Input & $\mathrm{H}^{+b}$ & $\frac{\text { Output }}{K / \mathrm{M}^{-1}}$ \\
\hline $\mathrm{UV}^{a}$ & 0 & $<5$ (low) \\
0 & 1 & $<5$ (low) \\
0 & 0 & 18 (low) \\
1 & 1 & 455 (high) \\
1 & ${ }^{a} 0=$ no UV; $1=50 \mathrm{~s} \mathrm{UV} \mathrm{exposure.}{ }^{b} 0=\mathrm{pH} 7 ; 1=\mathrm{pH} 6$. \\
\hline
\end{tabular}

flexibility in the design of tailor-made, $\mathrm{pH}$ sensitive, compounds. Efforts along these lines are undertaken in our laboratory.

In summary, we have presented a proof-of-principle study on a dually activated spiropyran derivative for $\mathrm{pH}$ - and light-controlled DNA-binding. The drug is only activated in the simultaneous events of UV exposure and a high local concentration of protons. We envision that the application of two complementary inputs - photonic and chemical-will have significant implications for general studies in this area, as the molecule is able to feel its chemical environment for sensory decision-making but must also be externally triggered using light.

This work was supported by the Swedish Research Council (VR), the European Research Council (ERC FP7/2007-2013 No. 203952), and the Research Foundations of Carl Trygger and Kristina Stenborg.

\section{Notes and references}

1 J. Andréasson and U. Pischel, Chem. Soc. Rev., 2010, 39, 174.

2 U. Pischel, Angew. Chem., Int. Ed., 2007, 46, 4026.

3 K. Szaciłowski, Chem. Rev., 2008, 108, 3481.

4 V. Balzani, A. Credi and M. Venturi, ChemPhysChem, 2003, 4, 49.

5 A. P. de Silva and N. D. McClenaghan, Chem.-Eur. J., 2004, 10, 574.

6 J. Andréasson, S. D. Straight, S. Bandyopadhyay, R. H. Mitchell, T. A. Moore, A. L. Moore and D. Gust, Angew. Chem., Int. Ed., 2007, 46, 958.

7 J. Andréasson, S. D. Straight, S. Bandyopadhyay, R. H. Mitchell, T. A. Moore, A. L. Moore and D. Gust, J. Phys. Chem. C, 2007, 111, 14274.

8 J. Andréasson, S. D. Straight, T. A. Moore, A. L. Moore and D. Gust, J. Am. Chem. Soc., 2008, 130, 11122.

9 A. P. de Silva and N. D. McClenaghan, J. Am. Chem. Soc., 2000, 122, 3965.

10 S. J. Langford and T. Yann, J. Am. Chem. Soc., 2003, 125, 11198.

11 S. J. Langford and T. Yann, J. Am. Chem. Soc., 2003, 125, 14951.

12 D. Margulies, G. Melman and A. Shanzer, Nat. Mater., 2005, 4, 768.

13 F. M. Raymo, Adv. Mater., 2002, 14, 401.

14 G. Strack, M. Ornatska, M. Pita and E. Katz, J. Am. Chem. Soc., 2008, 130, 4234

15 S. Ozlem and E. U. Akkaya, J. Am. Chem. Soc., 2009, 131, 48.

16 T. Konry and D. R. Walt, J. Am. Chem. Soc., 2009, 131, 13232.

17 R. J. Amir, M. Popkov, R. A. Lerner, C. F. Barbas III and D. Shabat, Angew. Chem., Int. Ed., 2005, 44, 4378.

18 S. Angelos, Y. W. Yang, N. M. Khashab, J. F. Stoddart and J. I. Zink, J. Am. Chem. Soc., 2009, 131, 11344.

19 S. Muramatsu, K. Kinbara, H. Taguchi, N. Ishii and T. Aida, J. Am. Chem. Soc., 2006, 128, 3764.

20 I. L. Cameron, N. K. R. Smith, T. B. Pool and R. L. Sparks, Cancer Res., 1980, 40, 1493.

21 J. A. Goode and D. J. Chadwick, The Tumor Microenvironment: Causes and Consequences of Hypoxia and Acidity, John Wiley \& Sons, Inc., New York, NY, 2001.

22 P. Montcourrier, P. H. Mangeat, C. Valembois, G. Salazar, A. Sahuquet, C. Duperray and H. Rochefort, J. Cell Sci., 1994, 107, 2381.

23 P. B. Dervan, Bioorg. Med. Chem., 2001, 9, 2215.

24 G. Mayer and A. Heckel, Angew. Chem., Int. Ed., 2006, 45, 4900.

25 J. Andersson, S. Li, P. Lincoln and J. Andréasson, J. Am. Chem. Soc., 2008, 130, 11836.

26 T. Stafforst and D. Hilvert, Chem. Commun., 2009, 287.

$27 \mathrm{pH} 6$ and pH 7 were chosen to reflect a typical difference of $c a .1$ $\mathrm{pH}$ unit between a cancer cell and a normal cell.

28 B. Nordén and F. Tjerneld, Biophys. Chem., 1976, 4, 191.

29 B. Nordén, M. Kubista and T. Kurucsev, Q. Rev. Biophys., 1992, $25,51$.

30 Contributions from electrostatic interactions between DNA and $\mathbf{1}$ cannot be excluded. These interactions, however, are very weak and typically not reflected in the absorption- or the LD spectra. 